\title{
COMPETITIVIDAD COMERCIAL EXTERIOR DE LAS ACTIVIDADES AGROALIMENTARIAS ANDALUZAS 2006-2014
}

\section{INTERNATIONAL TRADE COMPETITIVENESS OF ANDALUSIAN AGRIFOOD ACTIVITIES 2006-2014}

Belda Álvarez, José Ignacio (Universidad Pablo de Olavide) *

Morán Álvarez, Juan Carlos (Universidad de Sevilla) **

\section{RESUMEN}

En un mundo cada vez más globalizado, mirar al exterior se convierte en una salida para una economía que encuentra su mercado interior mermado por la crisis económica. Andalucía no ha sido una excepción y ha puesto su mirada en los mercados exteriores ante el colapso del interior durante el periodo de crisis.

Dado que el sector agroalimentario andaluz es uno de los más destacados de su economía, el presente artículo tiene como objetivo principal conocer el comportamiento de las exportaciones agroalimentarias andaluzas en relación con la UE-28 para el periodo 20062014. Para ello hemos tratado de descubrir el comportamiento de los sectores exportadores agroalimentarios intra y extra-UE (capítulos arancelarios 1 al 24), en función de su especialización exportadora y de su carácter competitivo o dinámico según la técnica shiftshare; comprobando su relación con la cuota de mercado.

Para alcanzar dicho objetivo hemos aplicado la técnica del análisis de los desplazamientos o análisis shift-share, y hallado la especialización y las cuotas de mercado.

Sabiendo que toda agregación disminuye el conocimiento y el detalle, hemos desagregado cuatro capítulos arancelarios hasta los cuatro dígitos, lo que nos ha permitido un conocimiento más detallado de cada uno de dichos sectores, comprobando, que, al desagregar, nos encontramos con subsectores cuyo comportamiento individual puede presentar diferencias más o menos importantes respecto al sector agregado.

En definitiva, un análisis de las exportaciones agroalimentarias andaluzas como el realizado nos permite mejorar el conocimiento del sector $\mathrm{y}$, en consecuencia, poder detectar las fortalezas y debilidades del mismo.

Palabras claves: Cuota de mercado, análisis shift-share, sector agroalimentario, competitivo, especialización exportadora. JEL: F10, F14.

\footnotetext{
ABSTRACT

In an increasingly globalized world, looking out becomes a way out for an economy that its domestic market has been eroded by the economic crisis. Andalusia hasn't been an exception and set its sights on foreign markets due to the collapse of its interior one during the crisis.

* Escuela Universitaria de Osuna, Edificio Antigua Universidad, Campo de Cipreses, 1, Apto. Correos 152, 41640, Osuna, Sevilla. joseiba@euosuna.org

* Departamento de Economía Aplicada III, Facultad de Ciencias Económicas y Empresariales, Avda. Ramón y Cajal, s/n, 41018, Sevilla. jcmoran@us.es

Recibido: Febrero de 2016. Aceptado: Mayo de 2016.
} 
Since the Andalusian agro-food sector is one of the highlights of its economy, this article's aim is "To know the behavior of Andalusian agro-food exports in relation to the EU-28 for 2006-2014 period". To do that we've tried to find out the behavior of EU and non-EU agrifood export sectors (tariff chapters 1 to 24), depending on their export specialization and its competitive and dynamic character using the "shift-share" technique; checking their relationship with the market share.

To achieve that goal we applied the analysis of displacement technique or shift-share analysis, and found the expertise and market shares.

Knowing that any aggregation reduces the knowledge and the detail, we have unbundled four tariff chapters to four digits, which has allowed us a detailed knowledge of each of those sectors, verifying that, by breaking down, there are subsectors whose Individual behavior may have differences, more or less significant, in the aggregate sector.

In short, an analysis of the Andalusian agro-food exports like the one done allows us to improve the knowledge about the sector and thus to be able to detect that the strengths and weaknesses.

Key words: Market share, shift - share analysis, Agri-food sector, competitive, export specialization. JEL: F10, F14.

\section{INTRODUCCIÓN}

Las economías española, en general, y andaluza, en particular, han dirigido sus esfuerzos a conquistar mercados exteriores que vinieran a suplir el descenso de las ventas en los mercados internos. Así, durante el periodo 2006 a 2014 y en términos corrientes, mientras las exportaciones intra-regionales de Andalucía descendían un 28,72 por 100 y las interregionales, aumentaban un 1,04 por 100 , las ventas internacionales crecían un 68,1 por 100 . Como consecuencia, la economía andaluza tiene un mayor grado de internacionalización: el peso de las exportaciones sobre el PIB ha pasado del 11 al 18,4 por 100, el grado de apertura se ha incrementado en 13,4 puntos porcentuales hasta alcanzar el 39,8 por 100; el número de empresas exportadoras creció un 22,8 por 100 , su facturación un 40,82 por 100 , mientras que las empresas exportadoras regulares crecieron un 15,82 por 100 y sus ventas un 42,42 por 100.

Al estudiar la economía de Andalucía, el sector agroalimentario ocupa un lugar preponderante, puesto que con datos provisionales de 2013, la agricultura, ganadería y silvicultura, tenía un peso en el PIB a precios corrientes del 4,6 por 100, mientras que el peso de la industria agroalimentaria fue del 2,1 por 100 . De forma conjunta ambas actividades tenían un peso en la economía andaluza del 6,7 por 100 en $2013^{1 .}$

Si centramos nuestra atención en el sector exterior, los principales datos quedan recogidos en el cuadro 1.

El protagonismo del sector agroalimentario en el conjunto de la economía andaluza se ve reflejado, también, en su evolución exterior, donde las empresas exportadoras del sector, regulares o no, suponen una parte importante del conjunto de empresas.

\footnotetext{
1 Datos del PIB de Andalucía: Contabilidad regional de Andalucía. Base 2010. Serie 1995/2014. Instituto de Estadística y Cartografía de Andalucía.

http://www.juntadeandalucia.es/institutodeestadisticaycartografia./craa/fich/craa1995-2014.xls.

Datos de comercio intra e interregional: Proyecto C-Intereg. (2015 a).

Datos sobre empresas: ICEX (2015 a)
} 
CUADRO 1: EMPRESAS EXPORTADORAS AGROALIMENTARIAS DE ANDALUCÍA, 2006-2014 (nº empresas y mill. €)

\begin{tabular}{|l|cccc|}
\hline & $\begin{array}{c}\text { \% X Andaluzas de. } \\
\text { Ind. Agro. }\end{array}$ & $\%$ X And, A, S, P*. & $\begin{array}{c}\% \text { X And vs Esp Ind. } \\
\text { Agro. }\end{array}$ & $\begin{array}{c}\% \text { X And vs Esp. } \\
\text { A, S, P*. }\end{array}$ \\
\hline Promedio $95 / 13$ & $22,3 \%$ & $7,9 \%$ & $13,9\left(1^{\circ}\right.$ posición $)$ & $15,54\left(2^{\circ}\right.$ posición $)$ \\
\hline
\end{tabular}

A,S,P: Agricultura, Silvicultura y Pesca.

Fuente: Proyecto C-Intereg. (2015 b y c)

\begin{tabular}{|c|c|c|c|c|c|}
\hline \multicolumn{6}{|c|}{$\begin{array}{l}\text { CUADRO 2: PERFIL DE LAS EMPRESAS EXPORTADORAS } \\
\text { AGROALIMENTARIAS DE ANDALUCÍA, 2006-2014 (no empresas y mill. } €)\end{array}$} \\
\hline & 2006 & 2014 & Var. 06/14 & $\begin{array}{c}\% \text { Sb Total } \\
2006\end{array}$ & $\begin{array}{c}\% \text { Sb Total } \\
2014\end{array}$ \\
\hline Agroalimentarias & 2.290 & 3.306 & 30,73 & 15,54 & 17,32 \\
\hline X. Agro. (mill. €) & 5.174 & 8.315 & 37,77 & 32,81 & 31,2 \\
\hline Agroalimentarias Regulares & 1.152 & 1.420 & 18,87 & 31,47 & 32,65 \\
\hline X. Reg. Agro (mill. €) & 4.509 & 7.400 & 39,07 & 34,09 & 32,21 \\
\hline
\end{tabular}

Por todo lo expuesto con anterioridad, el objetivo que nos hemos planteado en este trabajo es conocer el comportamiento competitivo de las exportaciones agroalimentarias andaluzas, teniendo como marco de referencia geográfico superior a la UE-28.

\section{METODOLOGÍA}

El método científico utilizado es el inductivo, al partir de las desagregaciones de las exportaciones agroalimentarias andaluzas mediante los veinticuatro primeros capítulos arancelarios, que hemos identificado como sectores exportadores agroalimentarios, usando de forma indistinta ambas denominaciones. Para ello hemos supuesto un comportamiento único para un capítulo arancelario, que está conformado por una serie de productos distintos y que, por lo tanto, pueden presentar un comportamiento heterogéneo.

En el trabajo hacemos una distinción entre exportaciones a la UE-28, o exportaciones intra-UE y las realizadas al resto del mundo o exportaciones extra-UE. Esto supone usar información estadística aduanera, para las exportaciones extra-UE, e información que proviene de encuestas en el caso intra-UE.

\subsection{Decisiones metodológicas}

El periodo de estudio elegido ha sido 2006 a 2014 con la idea de tener un ámbito temporal homogéneo de recesión o crecimiento de las variables a estudiar, con el fin de que al aplicar el análisis de los desplazamientos o análisis shift-share, los efectos no se anulen.

El nivel de desagregación de las exportaciones ha sido el de capítulos arancelarios del sistema de clasificación de mercancías conocido como Sistema Armonizado de Designación y Codificación de las Mercancías, o Sistema Armonizado (SA). En concreto, las exportaciones agroalimentarias vienen recogidas en los veinticuatro primeros. 
El problema que se nos plantea al desagregar por capítulos, es que en cada uno de ellos concurren bienes de distinta naturaleza, con lo cual su comportamiento en conjunto puede ser distinto al total de productos que lo componen, en función de que uno de ellos determine por sí solo el comportamiento del conjunto. Para poder realizar una primera valoración al respecto, en la parte final del trabajo, hemos aumentado el nivel de desagregación (partidas arancelarias) a cuatro capítulos, dos intra-UE y dos extra-UE.

El marco geográfico de referencia con el que hemos trabajado ha sido la UE-28, toda vez que casi el 60 por 100 de las exportaciones andaluzas de media durante el periodo analizado tienen ese destino. Si solo tenemos en cuenta las exportaciones agroalimentarias, la cifra asciende hasta, aproximadamente, el 80 por 100.

Hemos usado dos fuentes estadísticas. Para las exportaciones andaluzas hemos utilizado las base de datos ofrecidas por el ICEX España, Exportación e Inversión, a través de la herramienta ESTACOM que trabaja con los datos suministrados por la AEAT/ Departamento de Aduanas de la Agencia Tributaria de España.

Por lo que respecta a las exportaciones comunitarias, hemos trabajado con la Base de Datos de Comercio Internacional, elaborada por la Oficina Europea de Estadísticas (EUROSTAT).

Hemos tenido que trabajar con dos fuentes de datos estadísticas porque EUROSTAT aunque proporciona datos de todos los países de la Unión Europea, no lo hace de sus regiones. Por su parte, la base de datos ESTACOM ofrece datos de España, desagregándolos a nivel autonómico y provincial, pero no ofrece datos de la UE-28. Para poder realizar el análisis de los desplazamientos nos hemos visto obligados a trabajar con dos fuentes distintas, que no proporcionan datos armonizados de Andalucía y de la UE-28, y que tenían como nexo de unión las exportaciones de España, con valores diferentes en las fuentes.

Para poder armonizar los datos de ESTACOM y de EUROSTAT hemos realizado las siguientes operaciones:

1. Hemos calculado el peso de las exportaciones andaluzas en el conjunto de España para cada uno de los 24 capítulos arancelarios, con datos procedentes de ESTACOM.

$$
\left(X_{\text {iAnd.(Estacom) }} / X_{\text {iEsp.(Estacom) }}\right) * 100=y
$$

2. Los porcentajes calculados en el apartado anterior se aplicaban a los datos de exportaciones de España ofrecidos por EUROSTAT, para cada uno de los veinticuatro capítulos arancelarios, lo que nos permite obtener las exportaciones estimadas de Andalucía según datos EUROSTAT.

$$
y * X_{\text {iEsp.(Eurostat })}=X_{\text {iAnd.(Estimada Eurostat })}
$$

Estas estimaciones son las que hemos utilizado a lo largo del trabajo.

Para la desagregación de los capítulos en partidas arancelarias, y en aras a disponer de una misma estructuración de la nomenclatura de las mismas, hemos trabajado con ESTACOM y EUROSTACOM. Esta última, también del ICEX, utiliza como fuente la información proporcionada por EUROSTAT y se ha aplicado el mismo proceso de armonización de datos expuesto anteriormente.

En el análisis de la especialización y la competitividad de las exportaciones agroalimentarias andaluzas, hemos diferenciado entre exportaciones intra y extra-UE. 


\subsection{Indicadores y técnicas}

El análisis de la competitividad, en nuestro caso aplicado al sector agroalimentario, lo hemos realizado bajo el supuesto de que las exportaciones revelan capacidades productivas de una economía o del sector que estudiamos. Si nuestras ventas crecen más que las de nuestros competidores, esto significa que somos más competitivos.

Con este supuesto de partida, hemos utilizado dos indicadores estáticos de la posición competitiva como son el índice de especialización exportadora y la cuota de mercado; junto con un análisis shift-share que nos ha permitido identificar los sectores productivos agroalimentarios dinámicos y competitivos en los que estamos o no especializados, y su influencia en el crecimiento de las exportaciones, así como su repercusión en la evolución de la cuota de mercado, medida a través de la penetración real.

Es cierto que se trata de unas técnicas frecuentes en el análisis de competitividad exterior, y que hay otras técnicas como la técnica de Rasch que podría ser muy útil para medir el grado de penetración internacional de la agroalimentación andaluza o el índice de Herfindahl que puede ayudarnos a medir el grado de concentración o diversificación sectorial en nuestras exportaciones. En todo caso son las nuevas líneas con las que pretendemos completar el análisis que presentamos en este artículo.

A continuación describimos con algo más de detenimiento las técnicas utilizadas:

Índice de especialización exportadora (IEE):

Compara el tamaño relativo de un sector (capítulo arancelario) en una región o país, en nuestro caso Andalucía, con el tamaño relativo del mismo sector en un ámbito geográfico superior. En nuestro caso calculamos el IEE para los veinticuatro primeros capítulos arancelarios del SA, tomando como referencia la UE-28 y diferenciando entre comercio intra y extra-UE.

A partir de la definición de Fuentes e Hidalgo (1995) este índice podemos expresarlo de la siguiente manera:

$$
\mathrm{IE}_{i}=\left(\frac{X_{i}^{A N D A L U C I ́ A} / \sum_{i=1}^{n} X_{i}^{A N D A L U C I ́ A}}{X_{i}^{E U(28)} / \sum_{i=1}^{n} X_{i}^{U E(28)}}\right) \times 100
$$

Donde $X_{i}$ representa las exportaciones, intra-UE o extra-UE de cada uno de los veinticuatro capítulos arancelarios (sectores), que representan al sector agroalimentario, ya sean de Andalucía o de la UE-28. Un valor superior a 100 significa que las exportaciones del sector considerado tienen una presencia mayor en España que en el conjunto de la UE-28.

Cuota de mercado

A partir de la cuota de mercado calculamos la penetración real, que se expresa como la variación que tiene la cuota de mercado durante el periodo estudiado

$$
\mathrm{CM}_{i}=\left(\frac{X_{i}^{A N D A L U C I ́ A}}{X_{i}^{E U(28)}}\right) \times 100
$$


Donde $\mathrm{X}_{\mathrm{i}}$ representa las exportaciones de cada uno de los sectores exportadores, ya sean de Andalucía o de la UE-28, tanto intra-UE, como extra-UE. Calculamos la cuota para 2006 y 2014. La diferencia entre los valores obtenidos en 2006 y los de 2014 es lo que denominamos penetración real. Un valor positivo significará que hemos ganado cuota de mercado, mientras que uno negativo, que hemos perdido.

El análisis de los desplazamientos o análisis shift-share.

Se trata de una técnica estadística de análisis regional muy utilizada para estudiar la evolución de una variable económica en un territorio, teniendo como marco de referencia una entidad geográfica superior.

Como señala Rodríguez (2001): “Las descripciones más importantes de la técnica ShiftShare en su planteamiento clásico, corresponden a Creamer (1943), Duna (1960), Ashby (1964), Thirlwall (1967); Stiwell (1969 y 1970)".

La utilización de esta técnica para el estudio de la competitividad exportadora de una región viene avalada por el uso que de la misma han hecho autores como Green y Allaway (1985) y Coughlin y Pollard (2001), que han utilizado el análisis para evaluar la competitividad exportadora de las regiones de EEUU para productos concretos como Esteve et al. (2009).

La aplicación de los desplazamientos a las exportaciones españolas y regionales la podemos encontrar en distintos trabajos, entre otros, los de Alcántara y Blanes (2000); Donoso y Martín (2007); Seguí y Montserrat (2008); Esteve et al. (2009); Fernández y Márquez (2009); Gutiérrez (2012,2014), Myro et al. (2013) y Ruiz et al. (2014).

Ese análisis, se basa en la descomposición del crecimiento de la variable $\mathrm{X}$ en nuestro caso, las exportaciones intra o extra-UE andaluzas de cada uno de los sectores, en tres efectos distintos: nacional, competitivo y dinámico

La formulación del análisis de los desplazamientos queda, a partir de lo expresado por Martín y Martín (1993), de la siguiente manera:

$$
\mathrm{X}_{\mathrm{ia}}{ }^{*} \mathrm{~K}_{\mathrm{ia}}=\left(\mathrm{X}_{\mathrm{ia}}{ }^{*} \mathrm{~K}_{\mathrm{ue}}\right)+\mathrm{X}_{\mathrm{ia}}{ }^{*}\left(\mathrm{~K}_{\mathrm{ia}}-\mathrm{K}_{\mathrm{iue}}\right)+\mathrm{X}_{\mathrm{ia}}{ }^{*}\left(\mathrm{~K}_{\mathrm{iue}}-\mathrm{K}_{\mathrm{ue}}\right)
$$

Donde:

$\mathrm{X}_{\mathrm{ia}}$ : Exportaciones intra o extra-UE andaluzas para el capítulo $\boldsymbol{i}$ en 2006.

$\mathrm{K}_{\mathrm{ie}}$ : Tasa de crecimiento de las exportaciones intra o extra-UE andaluzas para el capítulo $\boldsymbol{i}$ (2006/2014).

$\mathrm{K}_{\mathrm{ue}}$ : Tasa de crecimiento del total de las exportaciones intra o extra-UE de la UE (2006/2014). $\mathrm{K}_{\text {iue }}$ : Tasa de crecimiento de las exportaciones intra o extra-UE de la UE para el capítulo i $(2006 / 2014)$.

Por lo tanto las expresiones matemáticas de los efectos son:

Efecto total: $\mathrm{X}_{\mathrm{ia}}{ }^{*} \mathrm{~K}_{\mathrm{ia}}$

Efecto nacional: $\left(\mathrm{X}_{\mathrm{ia}}{ }^{*} \mathrm{~K}_{\mathrm{ue}}\right)$

Efecto competitivo: $\mathrm{X}_{\mathrm{ia}}{ }^{*}\left(\mathrm{~K}_{\mathrm{ia}}-\mathrm{K}_{\mathrm{iue}}\right)$

Efecto dinámico: $\mathrm{X}_{\mathrm{ia}}{ }^{*}\left(\mathrm{~K}_{\mathrm{iue}}-\mathrm{K}_{\mathrm{ue}}\right)$.

Efecto nacional (EN): muestra la variación que ha experimentado el capítulo en Andalucía debido al crecimiento de las exportaciones en la UE-28 o, lo que es lo mismo, como resultado del arrastre ejercido por las exportaciones de la UE-28. Nos indica cuanto hubieran crecido las exportaciones del capítulo i, si su tasa de crecimiento hubiese sido igual a la experimentada por el total de exportaciones de la UE-28. 
Efecto competitivo (EC): nos muestra la competitividad del sector andaluz. Si dicho efecto es de signo positivo significa que el sector ha sido competitivo en Andalucía frente a la UE-28, debido a que las tasas de crecimiento del sector en Andalucía son superiores.

Efecto dinámico (ED): muestra qué parte de la variación producida en el capítulo se debe a un comportamiento dinámico del mismo en la UE-28. De modo que si el sector en la UE-28 evoluciona con una tasa de crecimiento mayor que la misma tasa para el conjunto de los sectores, produce un empuje positivo sobre el total. En ese caso tiene un valor positivo y provoca un aumento de las exportaciones del sector i en Andalucía.

En este artículo analizamos exclusivamente los efectos dinámico y competitivo, es decir, un estudio conjunto del dinamismo y la competitividad de cada uno de los sectores exportadores. Para ello, los sectores los hemos tipificado tal como hacen Gutiérrez et al. (1997): tipo 1, EC y ED positivos; tipo 2, EC negativo y ED positivo; tipo 3, EC positivo y ED negativo; tipo 4, EC y ED negativos.

De los cuatro tipos de sectores nos centramos en los sectores tipo 1, sobre todo si estamos especializados en ellos, puesto que al tener el EC positivo significa que ese sector presenta un mejor comportamiento competitivo en Andalucía que en la UE-28, y por tener un ED positivo, se trata de un sector que empuja el crecimiento de las exportaciones.

De forma subsidiaria podemos especializarnos en sectores tipo 2, puesto que si corregimos las causas que hacen que no sean competitivos, podrán clasificarse como sectores tipo 1.

El peor escenario es especializarse en los sectores tipo 3 y 4, es decir, en los que no somos competitivos, y en el caso del 4 se trata de un sector que no impulsa el crecimiento de las exportaciones.

Para finalizar con la metodología, y una vez descritas las técnicas de análisis que se ha utilizado, exponemos de forma resumida el esquema del trabajo. En primer lugar hemos calculado el IEE para conocer en qué sectores agroalimentarios estamos especializados y los hemos puesto en relación con la evolución de la cuota de mercado, medida a través de la penetración real. En este estudio hemos diferenciado el marco intra y extra-UE.

A continuación hemos aplicado el análisis de los desplazamientos a las exportaciones agroalimentarias andaluzas intra y extra-UE para detectar el comportamiento competitivo y dinámico de los sectores exportadores.

Por último, se analizan de forma conjunta los resultados de la especialización, cuota de mercado y desplazamientos, con el fin de detectar sectores exportadores agroalimentarios en los que estemos especializados con un buen comportamiento dinámico y competitivo (sectores tipo 1), puesto que mejoras en la especialización en sectores con un mal comportamiento competitivo y/o dinámico no es una buena señal para las perspectivas del sector exportador.

Para finalizar hemos elegido dos sectores exportadores intra-UE y dos extra-UE desagregándolos hasta cuatro cifras (partidas arancelarias), para comprobar si los comportamientos de los subsectores de desagregación se identifican con el sector agregado.

\section{3. ÍNDICE DE ESPECIALIZACIÓN EXPORTADORA (IEE) Y CUOTA DE MERCADO}

En este apartado analizamos el nivel y cambios en la especialización de las exportaciones agroalimentarias y los hemos puesto en relación con las variaciones en la cuota de mercado, medida esta última mediante la penetración real. 


\subsection{Comercio intra-UE}

En el periodo analizado no se han producido grandes cambios en la especialización de nuestro sector exportador ni desde un punto de vista cuantitativo ni cualitativo, toda vez que de doce sectores en los que estábamos especializados en 2006, pasamos a once en 2014; dejando de serlo los capítulos 5 y 17, y alcanzando dicha condición el 11. Además los sectores con más peso en la composición de las exportaciones intra-UE: 7, 15, 8, 2 y 22 son todos de especialización excepto el 2.

La nota negativa la encontramos en la pérdida de especialización por parte de dieciocho sectores, entre los que se encuentran los de mayor IEE en 2014. Sin embargo, esta reducción no ha significado, en todos los casos, perder posicionamiento en los mercados comunitarios, puesto que descensos importantes de especialización se han visto acompañados de mejoras importantes en la cuota de mercado, como le sucede al capítulo 15.

Las primeras conclusiones que podemos aportar sobre la especialización exportadora son:

1. Elevado IEE en los sectores en los que estamos especializados.

2. No hay una relación directa entre ganancias o pérdidas de cuota de mercado y nivel de especialización y cambios del mismo. No obstante, de los sectores que mejoran la misma, todos ganan cuota de mercado a excepción del 11.

Por todo lo señalado, podemos suponer que la estructura, orientación y protagonismo en destino de las exportaciones intra-UE no han sufrido apenas modificaciones entre $2006 \mathrm{y}$ 2014, lo que se traduce en un leve descenso del peso de las mismas en 0,23 puntos porcentuales, y un incremento en términos nominales de las exportaciones de un 52,9 por 100; desde los 4.635 millones de euros en 2006 a los 6.674 millones en 2014.

\begin{tabular}{|c|c|c|c|c|c|c|}
\hline \multicolumn{7}{|c|}{$\begin{array}{l}\text { CUADRO 3: CAPÍTULOS ARANCELARIOS ESPECIALIZADOS DE } \\
\text { LAS EXPORTACIONES INTRA-UE AGROALIMENTARIAS ANDALUZAS } 2014\end{array}$} \\
\hline & Var. IEE & IEE 2006 & IEE 2014 & $\begin{array}{l}\text { Penetración } \\
\quad \text { Real }\end{array}$ & $\begin{array}{l}\text { \% X Agro. } \\
2014\end{array}$ & $\begin{array}{l}\% \text { X. Tot. } \\
2014\end{array}$ \\
\hline 03 Pescados, crustáceos, moluscos. & $-56,10$ & 194,50 & 138,40 & 1,3134 & 1,76 & 0,77 \\
\hline $\begin{array}{l}06 \text { Plantas vivas; productos de } \\
\text { floricultura. }\end{array}$ & $-29,91$ & 158,63 & 128,72 & 0,0972 & 1,00 & 0,44 \\
\hline 07 Hortalizas, plantas alimenticias. & $-346,26$ & $2.836,10$ & $2.489,85$ & $-0,2305$ & 33,39 & 14,57 \\
\hline 08 Frutos / frutas comestibles. & $-79,36$ & $1.482,79$ & $1.403,43$ & 1,3005 & 23,03 & 10,05 \\
\hline 10 Cereales. & $-262,33$ & 397,46 & 135,13 & $-0,6356$ & 1,36 & 0,59 \\
\hline 11 Productos de la molinería, & 48,97 & 82,27 & 131,25 & $-0,7704$ & 0,40 & 0,17 \\
\hline $\begin{array}{l}12 \text { Semillas oleaginosas; plantas } \\
\text { industriales. }\end{array}$ & 86,45 & 113,33 & 199,78 & 0,2703 & 1,33 & 0.58 \\
\hline 14 Materias trenzables. & $-403,76$ & 622,87 & 219,11 & $-1,7840$ & 0,02 & 0,01 \\
\hline 15 Grasas, aceite animal o vegetal. & $-755,53$ & $2.780,04$ & $2.024,51$ & 5,1738 & 24,41 & 10,65 \\
\hline $\begin{array}{l}20 \text { Conservas verduras o fruta; } \\
\text { zumo. }\end{array}$ & $-38,63$ & 332,42 & 293,79 & $-1,6612$ & 3,97 & 1,73 \\
\hline $\begin{array}{l}22 \text { Bebidas todo tipo excepto } \\
\text { zumos. }\end{array}$ & $-116,18$ & 221,12 & 104,95 & $-0,0455$ & 2,47 & 1,08 \\
\hline
\end{tabular}




\subsection{Comercio extra-UE}

En el ámbito extra-UE se producen algunos cambios cuantitativos, pasamos de doce sectores de especialización a nueve. Desde una perspectiva cualitativa los cambios son mayores, dejando de estar especializados los capítulos 10,11, 14, "17. Azúcares: artículos de confitería" y "19. Productos de cereales, de pastelería", mientras que alcanzan dicha consideración en 2014 los capítulos 2 y 21. A pesar la reestructuración comentada, los más importantes en cuanto a peso en la composición de las ventas fuera de la Unión Europea, se mantienen como sectores de especialización.

Otro cambio importante que se produce es que, en líneas generales, aumentamos la especialización exportadora en productos agroalimentarios frente a los países no comunitarios, dado que de los veinticuatro capítulos que componen el sector, mejora el IEE en trece de ellos.

Las ganancias de especialización sí llevan aparejados aumentos de cuota de mercado, dado que de los quince sectores que ganan cuota, en trece de ellos mejora el IEE; son excepción a ello los capítulos 6 y 8.

\begin{tabular}{|c|c|c|c|c|c|c|}
\hline \multicolumn{7}{|c|}{$\begin{array}{l}\text { CUADRO 4: CAPÍTULOS ARANCELARIOS ESPECIALIZADOS DE LAS } \\
\text { EXPORTACIONES INTRA-UE AGROALIMENTARIAS ANDALUZAS } 2014\end{array}$} \\
\hline & Var. IEE & IEE 2006 & IEE 2014 & $\begin{array}{l}\text { Penetración } \\
\text { Real }\end{array}$ & $\begin{array}{l}\text { \% X Agro. } \\
2014\end{array}$ & $\begin{array}{l}\text { \% X. Tot. } \\
2014\end{array}$ \\
\hline $\begin{array}{l}02 \text { Carne y despojos } \\
\text { comestibles }\end{array}$ & 55,00 & 93,56 & 148,57 & 0,5544 & 4,30 & 0,70 \\
\hline $\begin{array}{l}03 \text { Pescados, crustáceos, } \\
\text { moluscos }\end{array}$ & 105,22 & 314,12 & 419,34 & 1,3134 & 5,28 & 0,86 \\
\hline $\begin{array}{l}05 \text { Otros productos de origen } \\
\text { animal }\end{array}$ & 95,84 & 107,34 & 203,18 & 0,8619 & 0,67 & 0,11 \\
\hline $\begin{array}{l}07 \text { Hortalizas, plantas } \\
\text { alimenticias }\end{array}$ & $-196,18$ & 600,26 & 404,08 & $-0,2305$ & 4,14 & 0,67 \\
\hline 08 Frutos / frutas comestibles & 100,83 & 323,46 & 424,29 & 1,3005 & 5,89 & 0,96 \\
\hline $\begin{array}{l}12 \text { Semillas oleaginosas; } \\
\text { plantas industriales }\end{array}$ & 11,01 & 103,95 & 114,95 & 0,2703 & 1,13 & 0,18 \\
\hline $\begin{array}{l}15 \text { Grasas, aceite animal o } \\
\text { vegetal }\end{array}$ & 184,79 & $2.084,58$ & $2.269,37$ & 5,1738 & 38,08 & 6,19 \\
\hline $\begin{array}{l}20 \text { Conservas verduras o } \\
\text { fruta; zumo }\end{array}$ & $-767,26$ & $1.941,37$ & $1.174,11$ & $-1,6612$ & 19,60 & 3,19 \\
\hline $\begin{array}{l}21 \text { Preparaciones } \\
\text { alimenticias diversas }\end{array}$ & 234,44 & 29,26 & 263,70 & 1,6719 & 7,25 & 1,18 \\
\hline
\end{tabular}

Los primeros apuntes que podemos aportar sobre la especialización exportadora son:

1. Mejora de la especialización y cuota de mercado en una parte importante de los capítulos del sector.

2. Cambios destacados en la composición de los capítulos en los que estamos especializados, hasta cinco, con pérdida de cuota de mercado para los mismos. 
Por todo lo señalado podemos suponer que la estructura, orientación y protagonismo en destino de las exportaciones extra-UE han sufrido modificaciones importantes entre 2006 y 2014, lo que le ha permitido aumentar en casi un punto porcentual su peso en la composición de las exportaciones extra-UE, que presenta para el periodo una tasa de crecimiento de algo más del 115 por 100 pasando de 888 millones de euros en 2006 a 1.909 millones en 2014.

\subsection{Comparación comercio intra y extra-UE}

De lo expuesto en los subepígrafes anteriores, podemos resaltar que existe un comportamiento diferenciado en la especialización por parte de los sectores exportadores intra y extra-UE. Las principales diferencias son:

1. El número de sectores especializados en 2014 es ligeramente mayor en el comercio intra que en el extra-UE (once frente a nueve). Por contra, en el marco extra-UE el número de sectores que mejoran su IEE es mayor (trece frente a seis). En ambos, quince capítulos mejoran su cuota de mercado.

2. Las mejoras de especialización se traducen en aumento de la cuota de mercado extra-UE-28, pero no sucede en todos los casos para las ventas intra-UE.

3. Se produce un cambio cualitativo en la especialización de mayor intensidad en el comercio extra-UE, pues cinco sectores pierden su condición de especializados y dos lo ganan, mientras que en el intra-UE dos pierden y uno gana.

4. El comportamiento competitivo del sector exportador ha sido más positivo a nivel extra que intra-UE, toda vez que es mayor el número de sectores de especialización, que mejoran su IEE y ganan cuota de mercado para el primero (siete), que para el segundo (uno). A esto hay que añadir que a nivel extra-UE, los esfuerzos en especialización, se traducen siempre en mejoras de cuota de mercado.

5. Comparando los sectores de especialización que coinciden en el comercio intra y extra-UE, vemos que alcanzan la cifra de seis, una cuarta parte de los que conforman el sector agroalimentario. Por lo que tenemos una amplia base común en la que sustentar nuestras ventas al exterior, ya sea dentro de la Unión Europea, como fuera de ella.

\section{RESULTADOS DEL ANÁLISIS DE LOS DESPLAZAMIENTOS O ANÁLISIS SHIFT-SHARE APLICADO A LAS EXPORTACIONES AGROALIMENTARIAS ANDALUZAS}

Hemos analizado la competitividad de las exportaciones agroalimentarias aplicando el análisis de los desplazamientos a las exportaciones intra y extra-UE correspondientes a los veinticuatro primeros capítulos arancelarios del sistema de clasificación de mercancías Sistema Armonizado, para el periodo 2006/2014, teniendo como entidad geográfica superior las UE-28.

En primer lugar, hemos analizado el comportamiento competitivo y dinámico de los sectores exportadores, para poder clasificar cada uno de ellos en las cuatro categorías que señalábamos en la metodología. Desde ese punto de partida, hemos cruzado los resultados con los obtenidos en el análisis de la especialización, para resaltar los sectores tipo 1, ambos efectos positivos, en los que estamos especializados.

Nuestro interés se centra en estos sectores, puesto que entendemos que hay que apostar por ellos como impulsores de las exportaciones y, por tanto, como creadores de riqueza y de empleo. En su defecto podemos apostar por los sectores tipo 2, efecto competitivo negativo y 
dinámico positivo. La especialización en estos sectores debe de llevar aparejado un trabajo por parte de los agentes económicos, tanto públicos como privados, con el fin de eliminar o disminuir las barreras que dificultan su competitividad y que puedan convertirse en un futuro en sectores tipo 1.

En el estudio se analiza por separado el comercio intra-UE y extra-UE, lo que nos permite detectar las diferencias de comportamiento que hay en ambas zonas geográficas.

A modo de introducción ofrecemos en el siguiente cuadro los principales resultado de los desplazamientos.

\section{CUADRO 5: RESULTADO DEL ANÁLISIS DE LOS DESPLAZAMIENTOS PARA EL COMERCIO INTRA Y EXTRA-UE 2006/2014 (mill. €)}

\begin{tabular}{|c|c|c|c|c|}
\hline & \multicolumn{2}{|c|}{ Exportaciones agroalimentarias } & \multicolumn{2}{|c|}{ Exportaciones totales } \\
\hline & Intra-UE & Extra-UE & Intra-UE & Extra-UE \\
\hline Efecto nacional & 720 & 423 & 1.642 & 2.7961 \\
\hline Efecto competitivo & 453 & 296 & 2.766 & 2.852 \\
\hline Efecto dinámico & 1.136 & 302 & 939 & 332 \\
\hline Variación de las exportaciones & 2.309 & 1.021 & 5.347 & 5.945 \\
\hline
\end{tabular}

Los datos que nos ofrece el cuadro 5 nos muestran la importancia del sector agroalimentario, sobre todo en nuestras ventas a la Unión Europea, puesto que suponen algo más del 43 por 100 del incremento de las exportaciones intra-UE, porcentaje que disminuye en el caso extra a algo más del 17 por 100. Por lo tanto, el sector agroalimentario centra su esfuerzo, principalmente en la Unión Europea, donde casi un 70 por 100 del aumento de sus exportaciones se generaron en este ámbito geográfico.

En cuanto al crecimiento de las ventas, para el conjunto del comercio, se origina principalmente por la competitividad de nuestros sectores exportadores, con mayor claridad en el ámbito intra-UE, que fuera de él. Por el contrario el aumento de las exportaciones agroalimentarias tiene su origen en el dinamismo de nuestros productos en el caso intra-UE, y en la evolución del sector exportador de la UE-28, o efecto nacional, en el caso extra-UE.

\subsection{Comercio intra-UE}

El cuadro 6 nos muestra los principales resultados que aporta el análisis de los desplazamientos sobre la competitividad y dinamismo de las exportaciones agroalimentarias andaluzas dentro de las fronteras comunitarias.

\begin{tabular}{|c|c|c|c|c|c|c|c|c|}
\hline \multicolumn{9}{|c|}{$\begin{array}{l}\text { CUADRO 6: EFECTOS COMPETITIVO Y DINÁMICO DE } \\
\text { LAS EXPORTACIONES AGROALIMENTARIAS, 2006-2014 }\end{array}$} \\
\hline & $\begin{array}{l}\mathrm{N}^{\circ} \text { Cap. } \\
(+)\end{array}$ & $\begin{array}{l}\text { Aportación } \\
\text { incremento } \\
X .(\text { Mill. } €)\end{array}$ & $\begin{array}{l}\% \times \\
\text { Agro. } \\
2014\end{array}$ & $\begin{array}{l}\% \text { X. } \\
\text { Tot. } \\
2014\end{array}$ & $\begin{array}{l}\mathrm{N}^{\circ} \text { Cap. } \\
(-)\end{array}$ & $\begin{array}{l}\text { Restan al } \\
\text { incremento } \\
\text { X. (Mill. } € \text { ) }\end{array}$ & $\begin{array}{l}\% \mathrm{X} \\
\text { Agro. } \\
2014\end{array}$ & $\begin{array}{l}\% \text { X. } \\
\text { Tot. } \\
2014\end{array}$ \\
\hline Efecto competitivo & 12 & 804 & 68,09 & 29,71 & 12 & 351 & 31,91 & 13,92 \\
\hline Efecto dinámico & 23 & 1.138 & 99,00 & 43,19 & 1 & 1,3 & 1,00 & 0,44 \\
\hline
\end{tabular}


Hemos comentado que en el ámbito intra-UE el aumento de las exportaciones venía dado por comportamiento dinámico de las mismas. El cuadro anterior nos lo muestra de forma clara, toda vez que de los veinticuatro sectores estudiados, veintitrés presentan un comportamiento dinámico y los no dinámicos apenas si restan 1,3 millones de euros al crecimiento de las exportaciones. Por el contrario, la falta de competitividad de doce sectores nos resta 351 millones de crecimiento a las mismas.

Los datos expuestos suponen que una parte importante, en concreto veintitrés, de los sectores agroalimentarios vamos a poder clasificarlos como tipo 1 y 2 , tal como nos muestra el cuadro 7.

\begin{tabular}{|c|c|c|c|c|c|c|c|}
\hline \multicolumn{8}{|c|}{$\begin{array}{c}\text { CUADRO 7: DISTRIBUCIÓN DE LOS SECTORES AGROALIMENTARIOS } \\
\text { POR TIPO Y APORTACIÓN AL SECTOR }\end{array}$} \\
\hline & $\mathrm{N}^{\mathrm{o}}$ Sectores & $\begin{array}{l}\text { Efecto } \\
\text { Nacional } \\
\text { (Mill. } €)\end{array}$ & $\begin{array}{l}\text { Efecto } \\
\text { Competitivo } \\
\text { (Mill. } €)\end{array}$ & $\begin{array}{l}\text { Efecto } \\
\text { Dinámico } \\
\text { (Mill. } € \text { ) }\end{array}$ & $\begin{array}{l}\text { Variación } \\
X(\text { Mill. } €)\end{array}$ & $\begin{array}{l}\text { \% X Agro. } \\
2014\end{array}$ & $\begin{array}{c}\text { \% X. Tot. } \\
2014\end{array}$ \\
\hline Tipo 1 & 11 & 455 & 799 & 469 & 1.723 & 67,09 & 29,27 \\
\hline Tipo 2 & 12 & 257 & -351 & 669 & 574 & 31,91 & 13,92 \\
\hline Tipo 3 & 1 & 9 & 4 & -1 & 12 & 1,00 & 0,44 \\
\hline Tipo 4 & - & - & - & - & - & & - \\
\hline
\end{tabular}

La distribución de los sectores por tipos que refleja el cuadro anterior, con ningún sector tipo 4 y un solo tipo 3, nos muestra un comportamiento del sector exportador excelente y con perspectivas de futuro, siempre y cuando eliminemos las barreras que frenan la competitividad de los sectores 2 y así poder convertirlos en sectores tipo 1, con lo que en el mejor de los casos podían haber aumentado las exportaciones intra-UE en 351 millones de euros.

En este punto nos preguntamos ¿estamos apostando por este tipo de sectores, en especial los tipo 1 ?

Para contestar a esta pregunta, el cuadro 8 muestra los sectores clasificados como tipo 1, resaltando aquellos en los que estamos especializados, pues son estos últimos los que más nos interesa detectar.

De los once sectores tipo 1, solo estamos especializados en cinco, pero son los más destacados, con una aportación al crecimiento de las exportaciones por el EC de 714 millones de euros (799 aportaban el total de sectores tipo 1) y de 416 por el ED, (469). De estos cinco destacan el 7 y el 8, que ocupan la primera y la tercera posición de los capítulos arancelarios más exportadores intra UE-28 con casi un 25 por 100 del total de ventas a la UE-28. Si sumamos el capítulo " 15 . Grasas, aceite animal o vegetal" que está clasificado como tipo 2, y que ocupa la segunda posición, tenemos algo más de un tercio de las ventas a la comunidad y una aportación a las exportaciones cercana a los dos mil millones de euros.

A pesar del buen comportamiento de los sectores de especialización tipo 1, podemos observar como tres de ellos, 7,11 y 20, pierden cuota de mercado, medida a través de la penetración real. Por el contrario, el resto de sectores tipo 1, aunque no sean de especialización, si consiguen mejorar la cuota intra-UE, aunque de forma leve. 


\begin{tabular}{|c|c|c|c|c|c|c|c|c|}
\hline \multicolumn{9}{|c|}{ CUADRO 8: SECTORES TIPO 1 DE LAS EXPORTACIONES INTRA-UE } \\
\hline & $\begin{array}{l}\text { Var. } \\
\text { IEE }\end{array}$ & $\begin{array}{l}\text { IEE } \\
2014\end{array}$ & $\begin{array}{l}\text { Penetración } \\
\text { Real }\end{array}$ & $\mathrm{EN}^{*}$ & $\mathrm{EC}^{*}$ & ED* & $\begin{array}{c}\% \text { X } \\
\text { Agro. } \\
2014\end{array}$ & $\begin{array}{c}\% \text { X. } \\
\text { Tot. } \\
2014\end{array}$ \\
\hline 01 Animales vivos & 1,80 & 28,49 & 0,1065 & 1,08 & 3,57 & 1,11 & 0,18 & 0,08 \\
\hline $\begin{array}{l}02 \text { Carne y despojos } \\
\text { comestibles }\end{array}$ & $-7,14$ & 92,30 & 0,5544 & 16,17 & 31,07 & 24,05 & 2,54 & 1,11 \\
\hline $\begin{array}{l}07 \text { Hortalizas, plantas } \\
\text { alimenticias }\end{array}$ & $-346,26$ & $2.489,85$ & $-0,2305$ & 249,40 & 304,86 & 163,15 & 33,39 & 14,57 \\
\hline $\begin{array}{l}\text { 08 Frutos / frutas } \\
\text { comestibles }\end{array}$ & $-79,36$ & $1.403,43$ & 1,3005 & 146,83 & 306,40 & 194,27 & 23,03 & 10,05 \\
\hline $\begin{array}{l}09 \text { Café, té, Yerba mate y } \\
\text { especias }\end{array}$ & 21,97 & 43,28 & 0,2385 & 0,42 & 9,70 & 2,82 & 0,23 & 0,10 \\
\hline $\begin{array}{l}11 \text { Productos de la } \\
\text { molinería }\end{array}$ & 48,97 & 131,25 & $-0,7704$ & 1,15 & 13,90 & 4,44 & 0,40 & 0,17 \\
\hline $\begin{array}{l}12 \text { Semillas oleaginosas; } \\
\text { plantas industriales }\end{array}$ & 86,45 & 199,78 & 0,2703 & 3,06 & 50,71 & 16,64 & 1,33 & $\mathbf{0 , 5 8}$ \\
\hline $\begin{array}{l}20 \text { Conservas verduras } \\
\text { o fruta; zumo }\end{array}$ & $-38,63$ & 293,79 & $-1,6612$ & 26,79 & 37,72 & 37,81 & 3,97 & 1,73 \\
\hline $\begin{array}{l}21 \text { Preparaciones } \\
\text { alimenticias diversas }\end{array}$ & 18,41 & 64,08 & 1,6719 & 3,10 & 26,26 & 8,96 & 0,86 & 0,37 \\
\hline $\begin{array}{l}23 \text { Residuos industria } \\
\text { alimentaria }\end{array}$ & $-9,57$ & 41,65 & $-0,0947$ & 3,07 & 2,52 & 12,86 & 0,56 & 0,24 \\
\hline $\begin{array}{l}24 \text { Tabaco y sus } \\
\text { sucedáneos }\end{array}$ & 6,88 & 71,88 & 0,0711 & 3,56 & 12,75 & 2,61 & 0,61 & 0,26 \\
\hline
\end{tabular}

Dado que en los sectores en los que estamos especializados, el IEE supera con creces el valor de 100, en especial en el 7 y 8, podemos pensar que seguiremos especializados en estos mismos en un futuro. Similar razonamiento podemos hacer en el resto los tipo 1, los cuales, con valores del IEE muy alejados de 100, a excepción del capítulo 2, no esperamos que en el futuro se conviertan en sectores de especialización.

Del resto de sectores de especialización clasificados como tipo 2, a saber 15, 14, 3, 10 y 22 , dos ganan cuota de mercado. Todos ellos disminuyen su IEE. Por último, el 6, clasificado tipo 3, mejora su cuota de mercado y empeora su nivel de especialización.

Del análisis conjunto para el comercio intra-UE de la especialización, penetración real y análisis de los desplazamientos, podemos adelantar algunas conclusiones:

1. El número de sectores tipo 1, en el que estamos especializado, cinco, supone la mitad de los mismos, pero entre ellos se encuentran dos de los más exportadores de Andalucía, con casi una cuarta parte de las mismas. Además el IEE de estos sectores es alto y mantienen la especialización en el periodo estudiado, a excepción del 11, productos de la molinería, que no era un sector de especialización en 2006, pero si en 2014. Con todo ello podemos pensar que es posible consolidar alrededor del 25 por 100 de las exportaciones intra-UE en 
sectores de especialización, competitivos y dinámicos, con lo que supone de mantenimiento o aumento de las exportaciones en el futuro.

2. La especialización en sectores tipo 1 garantiza un aumento sostenido de las exportaciones, pero no asegura un crecimiento de la cuota de mercado, ya que de los cinco sectores clasificados en este grupo, tres de ellos, 7, 11 y 20 pierden cuota de mercado.

3. Los sectores tipo 2, en los cuales podemos apostar, de forma subsidiaria a los tipo 1, siempre y cuando trabajemos en la línea de mejorar su competitividad, aportan una cuarta parte del incremento de las ventas intra-UE de productos agroalimentarios, con un peso sobre las exportaciones intra-UE del 13,92 por 100 . La especialización en todos ellos disminuye, lo que hace que pasemos de siete sectores de especialización en 2006 a cinco en 2014, de los cuales tres pierden cuota de mercado.

4. Como nota positiva, solo tenemos un sector tipo 3 y ningún sector tipo 4 dentro de los agroalimentarios.

5. En su conjunto, el sector exportador agroalimentario pierde nivel de especialización en dieciocho de los veinticuatro capítulos arancelarios que lo componen, a pesar de lo cual solo pierden la condición de especializados dos sectores, el "5. Otros productos de origen animal" y el "17. Azúcares: artículos de confitería”.

6. No hay grandes cambios en los productos en los que estamos especializados, pues solo un nuevo sector, "11. Productos de la molinería", alcanza un IEE superior a 100 en 2014, y dos sectores que pierden su condición de especializados, tal y como hemos señalado anteriormente.

7. Las ganancias de cuota de mercado están más relacionadas con el dinamismo de los sectores que con su competitividad o especialización.

\subsection{Comercio extra-UE}

El cuadro 9 nos muestra los principales resultados que aporta el análisis de los desplazamientos sobre la competitividad y dinamismo de las exportaciones agroalimentarias andaluzas fuera de la Unión Europea.

\begin{tabular}{|c|c|c|c|c|c|c|c|c|}
\hline \multicolumn{9}{|c|}{$\begin{array}{l}\text { CUADRO 9: EFECTOS COMPETITIVO Y DINÁMICO DE } \\
\text { LAS EXPORTACIONES AGROALIMENTARIAS, 2006-2014 }\end{array}$} \\
\hline & $\begin{array}{c}\mathrm{N}^{\circ} \text { Cap. } \\
(+)\end{array}$ & $\begin{array}{l}\text { Aportación } \\
\text { incremento } \\
\text { X. (Mill. } \\
€)\end{array}$ & $\begin{array}{l}\% \times \\
\text { Agro. } \\
2014\end{array}$ & $\begin{array}{l}\% \times \text { X. } \\
\text { Tot. } \\
2014\end{array}$ & $\begin{array}{c}\mathrm{N}^{\circ} \text { Cap. } \\
(-)\end{array}$ & $\begin{array}{c}\text { Restan al } \\
\text { incremento } \\
\text { X. (Mill. } \\
€)\end{array}$ & $\begin{array}{l}\% \mathrm{X} \\
\text { Agro. } \\
2014\end{array}$ & $\begin{array}{l}\% \text { X. } \\
\text { Tot. } \\
2014\end{array}$ \\
\hline $\begin{array}{c}\text { Efecto } \\
\text { competitivo }\end{array}$ & 15 & 552 & 65,56 & 10,66 & 9 & 256 & 34,44 & 5,6 \\
\hline $\begin{array}{c}\text { Efecto } \\
\text { dinámico }\end{array}$ & 20 & 325 & 99,05 & 16,11 & 4 & 23 & 0,95 & 0,15 \\
\hline
\end{tabular}

En el caso del comercio extra-UE, el efecto competitivo es el que marca la evolución del sector, puesto que aunque son menos los capítulos competitivos que dinámicos, y su peso 
también es menor, la aportación de los primeros al incremento de las exportaciones con 552 millones de euros es mayor que la realizada por los capítulos dinámicos, 325 millones.

En el caso de los sectores con EC y ED negativos, los primeros son más, 9 frente a 4, con mayor peso, 5,6 por 100 y 0,15 por 100 respectivamente, y restan más a las ventas extraUE, 256 millones frente a 23. Por todo ello, la competitividad o su falta es la que determina en gran medida el comportamiento del sector agroalimentario fuera de la UE-28.

\begin{tabular}{|c|c|c|c|c|c|c|c|}
\hline \multicolumn{8}{|c|}{$\begin{array}{l}\text { CUADRO 10: DISTRIBUCIÓN DE LOS SECTORES AGROALIMENTARIOS } \\
\text { POR TIPO Y APORTACIÓN AL SECTOR }\end{array}$} \\
\hline & $\mathrm{N}^{\mathrm{o}}$ sectores & $\begin{array}{c}\text { Efecto } \\
\text { nacional } \\
(\text { mill. } €)\end{array}$ & $\begin{array}{l}\text { Efecto } \\
\text { competitivo } \\
\text { (mill. } € \text { ) }\end{array}$ & $\begin{array}{c}\text { Efecto } \\
\text { dinámico } \\
(\text { mill. } €)\end{array}$ & $\begin{array}{c}\text { Variación } \\
X(\text { mill. } €)\end{array}$ & $\begin{array}{c}\text { \% X Agro. } \\
2014\end{array}$ & $\begin{array}{c}\text { \% X. Tot. } \\
2014\end{array}$ \\
\hline Tipo 1 & 13 & 179 & 546 & 136 & 861 & 64,74 & 10,53 \\
\hline Tipo 2 & 7 & 226 & -235 & 186 & 180 & 34,31 & 5,58 \\
\hline Tipo 3 & 2 & 4 & 6 & -3 & 7 & 0,82 & 0,13 \\
\hline Tipo 4 & 2 & 14 & -21 & -20 & -27 & 0,13 & 0,02 \\
\hline
\end{tabular}

Al igual que sucedía en el comercio intra-UE, los sectores tipo 1, con trece, y los sectores tipo 2, con siete, influyen de forma determinante en la evolución del sector.

Son fundamentales los sectores tipo 1, que tienen una influencia en el crecimiento de las exportaciones de casi 900 millones de euros y suponen el 10,53 por 100 del total de las mismas.

Dentro de este grupo, estamos especializados en siete de ellos, con una aportación al crecimiento de las exportaciones de 831 millones de euros. Su elevado nivel de especialización, en el periodo analizado en todos ellos el IEE crece y ganan cuota de mercado, nos hace pensar que en estos productos tenemos una base de futuro crecimiento de las exportaciones.

No obstante, el capítulo 15 es el que monopoliza el comportamiento de las exportaciones extra-UE de productos agroalimentarios, ya que como sector tipo 1 es el que alcanza mayor nivel de especialización, su aportación al incremento de las exportaciones vía EC, ED y EN son las mayores de todos los capítulos que conforman el sector. Por último, su peso sobre el conjunto de las exportaciones extra-UE andaluzas del 6,19 por 100, lo convierten en el sector de mayor importancia en el agroalimentario y el quinto del conjunto de capítulos arancelarios, por detrás del "27. Combustibles; aceites minerales", "99. Otros productos", "88. Aeronaves, vehículos espaciales" y "74. Cobre y sus manufacturas".

Para terminar, tal como hicimos en el apartado anterior, comparamos los resultados de especialización, penetración real y análisis de los desplazamientos.

En el ámbito extra-UE, trece sectores incrementan su nivel de especialización, coincidiendo casi en su totalidad con los sectores tipo 1. El sector "24. Tabaco y sus sucedáneos", mejora el IEE, pero es un sector tipo 3; y el sector "18. Cacao y sus preparaciones", que se clasificó como tipo 1, pero empeoró su IEE. Dentro del grupo de sectores tipo 1, siete son de especialización, con el protagonismo para el 15 como señalamos anteriormente.

Todos los sectores que mejoran su especialización, aumentan su cuota de mercado a excepción del 18 y el "6. Plantas vivas; productos de la floricultura" que aumenta su cuota, 
pero reducen su IEE. El resto de sectores que lo empeoran, también pierden cuota de mercado.

\begin{tabular}{|c|c|c|c|c|c|c|c|c|}
\hline \multicolumn{9}{|c|}{ CUADRO 11: SECTORES TIPO 1 DE LAS EXPORTACIONES EXTRA-UE } \\
\hline & $\begin{array}{l}\text { Var. } \\
\text { IEE }\end{array}$ & $\begin{array}{l}\text { IEE } \\
2014\end{array}$ & $\begin{array}{l}\text { Penetración } \\
\text { Real }\end{array}$ & $\mathrm{EN}^{*}$ & $\mathrm{EC} *$ & ED* & $\begin{array}{c}\% \text { X } \\
\text { Agro. } \\
2014\end{array}$ & $\begin{array}{c}\% \text { X. } \\
\text { Tot. } \\
2014\end{array}$ \\
\hline 01 Animales vivos & 13,12 & 21,66 & 0,1065 & 0,19 & 2,05 & 0,23 & 0,15 & 0,02 \\
\hline $\begin{array}{l}\text { 02 Carne y despojos } \\
\text { comestibles }\end{array}$ & 55,00 & 148,57 & 0,5544 & 8,16 & 44,44 & 12,42 & 4,30 & $\mathbf{0 , 7 0}$ \\
\hline $\begin{array}{l}03 \text { Pescados, crustáceos, } \\
\text { moluscos }\end{array}$ & 105,22 & 419,34 & 1,3134 & 15,40 & 45,75 & 7,28 & 5,28 & $\mathbf{0 , 8 6}$ \\
\hline $\begin{array}{l}04 \text { Leche, productos } \\
\text { lácteos; huevos }\end{array}$ & 13,25 & 19,49 & 0,1031 & 0,75 & 11,15 & 1,08 & 0,76 & 0,12 \\
\hline $\begin{array}{l}05 \text { Otros productos de } \\
\text { origen animal }\end{array}$ & 95,84 & 203,18 & 0,8619 & 1,25 & 7,92 & 1,10 & 0,67 & 0,11 \\
\hline $\begin{array}{l}\text { 08 Frutos / frutas } \\
\text { comestibles }\end{array}$ & 100,83 & 424,29 & 1,3005 & 14,94 & 49,99 & 16,22 & 5,89 & 0,96 \\
\hline $\begin{array}{l}09 \text { Café, té, Yerba mate y } \\
\text { especias }\end{array}$ & 24,94 & 60,45 & 0,2385 & 0,85 & 4,30 & 0,59 & 0,39 & 0,06 \\
\hline $\begin{array}{l}12 \text { Semillas oleaginosas; } \\
\text { plantas industriales }\end{array}$ & 11,01 & 114,95 & 0,2703 & 2,95 & 7,38 & 5,12 & 1,13 & 0,18 \\
\hline $\begin{array}{l}13 \text { Jugos y extractos } \\
\text { vegetales }\end{array}$ & 23,60 & 37,55 & 0,1889 & 0,20 & 1,76 & 0,04 & 0,13 & 0,02 \\
\hline $\begin{array}{l}15 \text { Grasas, aceite animal } \\
\text { o vegetal }\end{array}$ & 184,79 & $2.269,37$ & 5,1738 & 128,28 & 240,26 & 89,15 & 38,08 & 6,19 \\
\hline $\begin{array}{l}16 \text { Conserva de carne o } \\
\text { pescado }\end{array}$ & 11,49 & 77,92 & 0,2035 & 1,58 & 3,16 & 0,30 & 0,44 & 0,07 \\
\hline $\begin{array}{lccc}18 & \text { Cacao } & \text { y } & \text { sus } \\
\text { preparaciones } & & \end{array}$ & $-1,70$ & 15,60 & 0,0206 & 0,88 & 0,97 & 1,35 & 0,26 & 0,04 \\
\hline $\begin{array}{l}21 \quad \text { Preparaciones } \\
\text { alimenticias diversas }\end{array}$ & 234,44 & 263,70 & 1,6719 & 3,14 & 127,24 & 1,45 & 7,25 & 1,18 \\
\hline
\end{tabular}

Del análisis conjunto que acabamos de realizar podemos apuntar las siguientes conclusiones:

1. El número de sectores tipo 1 en los que estamos especializados es alto, destacando sobremanera el capítulo 15. Todos ellos presentan un excelente IEE, mejoran la especialización y ganan cuota de mercado, lo que nos indica que estamos ante un grupo de productos que pueden ser una base estable para el crecimiento de las exportaciones extra-UE.

2. Solo cuatro sectores se clasificaron como sectores tipo 3 y 4 , y apenas superan el 1 por 100 de las exportaciones. 
3. Solo tenemos dos sectores nuevos de especialización: "21. Preparaciones alimenticias diversas" y "2. Carne y despojos comestibles", ambos sectores tipo 1. Por el contrario, perdemos la especialización en cinco sectores. Por lo tanto, parece que se está produciendo un cambio productivo ante la disminución de productos en los que nos especializamos, mientras que se mantiene un núcleo, con visos de estabilidad toda vez que mejoran su nivel de especialización.

4. La ganancia de cuota de mercado está directamente relacionada con productos competitivos y de mejora de la especialización.

5. Los sectores tipo 2, a los que debemos prestar atención, de forma subsidiaria a los sectores tipo 1, tienen protagonismo en el conjunto del sector con siete capítulos, un 5,58 por 100 de las exportaciones y una aportación a las ventas extra-UE de 180 millones de euros.

\subsection{Comparación comercio intra y extra-UE}

Dado que estamos estudiando dos ámbitos geográficos de actuación, las exportaciones a la UE-28 y las ventas al resto del mundo, terminamos el análisis que hemos realizado en los epígrafes anteriores, ofreciendo una comparativa de los resultados obtenidos en ambas zonas geográficas, mostrando las diferencias en el comportamiento competitivo y dinámico de los sectores exportadores agroalimentarios.

En primer lugar hay que señalar que el número de sectores con EC positivo es mayor para el comercio extra que para el intra-UE (15 frente a 12), mientras que el dinamismo es protagonista en el comercio intra-UE (23) frente al extra-UE (20). Esta diferencia queda también reflejada en su aportación al aumento de las ventas internacionales. Para el comercio intra-UE, los sectores competitivos aportaron 804 millones de euros frente a los 1.138 de los sectores dinámicos; mientras que en el comercio extra-UE, la aportación de los sectores competitivos es mayor que la realizada por los dinámicos, 552 millones de los primeros, frente a 325 de los segundos.

En segundo lugar, debemos señalar que la mejora en los niveles de especialización ha surtido mayores efectos en el comercio extra-UE que en el intra-UE, a partir de su aportación al aumento de las exportaciones vía EC y ED. Como podemos observar en el cuadro 12, los sectores de especialización aportan 1.472 millones de euros en el comercio intra-UE, por 651 del extra-UE; ahora bien si tenemos en cuenta los sectores que mejoran su IEE, la aportación en el primer caso es de 154 millones y en el segundo 683.

Podemos también observar como se ha realizado un mayor esfuerzo de especialización en el comercio extra-UE, puesto que los sectores de especialización que mejoran la misma en el comercio intra-UE aportaron 86 millones, frente a los 656 del primero.

Centrándonos en los sectores tipo 1 en los que estamos especializados, que son los que tenían nuestra atención prioritaria, podemos observar como la aportación de estos sectores en el comercio intra-UE ha sido de mayor envergadura que en el extra-UE, 1.130 millones frente a 656, aunque eran menos los sectores en el primer caso (cinco) que en el segundo (siete). Cara al futuro puede que esta situación revierta ya que en el comercio intra-UE tres disminuyen su IEE, mientras que en el extra-UE todos los tipo 1 todos mejoran la especialización, que lleva aparejada un aumento de cuota de mercado. 


\section{CUADRO 12: APORTACIÓN VÍA COMPETITIVIDAD Y DINAMISMO DE LOS} SECTORES EXPORTADORES SEGÚN TIPO Y ESPECIALIZACIÓN (mill. €)

\begin{tabular}{|l|ccc|ccc|}
\hline & \multicolumn{3}{|c|}{ Intra-UE } & \multicolumn{3}{c|}{ Extra-UE } \\
\hline \multirow{2}{*}{ Aportación sectores de especialización } & EC & ED & EC+ED & EC & ED & EC+ED \\
\cline { 2 - 8 } Aportación sectores que mejoran IEE & 433 & 1.039 & 1.472 & 440 & 211 & 651 \\
Aportación sectores de especialización que mejoran & 117 & 37 & 154 & 549 & 134 & 683 \\
IEE & 65 & 21 & 86 & 523 & 133 & 656 \\
Aportación sectores de especialización Tipo 1 & 714 & 416 & 1.130 & 523 & 133 & 656 \\
Aportación sectores de especialización Tipo 2 & -285 & 624 & 339 & -83 & 78 & -5 \\
Aportación sectores de especialización Tipo 3 & 4 & -1 & 3 & - & - & - \\
Aportación sectores de especialización Tipo 4 & - & - & - & - & - & - \\
\hline Fuente: Elaboración propia a partir de los datos de ICEX (2015 b) y EUROSTAT (2015) & & \\
\hline
\end{tabular}

No obstante lo señalado, el comercio intra-UE tiene interesantes opciones de crecer en el futuro basado en los sectores tipo 2 de especialización, siempre y cuando mejoren los niveles de competitividad de los mismos. Para el comercio intra-UE, su número es mayor, cinco frente a dos, y el nivel de aportación al incremento de las exportaciones muy diferente, con 339 millones en el primer caso, y un descenso de 5 en el segundo. El único pero que podemos poner a la situación de los tipo 2 intra-UE, es que en todos ellos descienden su IEE, y tres de ellos pierden cuota de mercado.

Por último, como elemento positivo señalar que para ambas zonas geográficas de comercio, no estamos especializados en sectores tipo 3 y 4, toda vez que habíamos remarcado que no es una situación con perspectivas positivas para el futuro de las exportaciones estar especializado en algunos de estos dos sectores.

\subsection{Análisis desagregado a partidas arancelarias}

Una vez finalizado el análisis de los veinticuatro capítulos arancelarios que conforman el sector agroalimentario, hemos desagregado cuatro, al siguiente nivel; es decir, a partidas arancelarias, lo que nos ha permitido comprobar si la agregación distorsiona los resultados obtenidos a nivel de capítulos, y si el conjunto de las partidas arancelarias que conforman un capítulo se comporta de forma similar al mismo, o por el contrario, el comportamiento del capítulo viene determinado por el que experimenta una de ellas.

Por último, al desagregar hemos querido conocer un poco más en profundidad algunos sectores que nos han parecido interesantes o determinantes en el comportamiento del sector exportador agroalimentario intra y extra-UE.

En el caso del comercio intra-UE, los sectores desagregados han sido el 8. "Frutas y Frutos comestibles" y el "15. Grasas animales o vegetales y aceites productos de su desdoblamiento; grasas alimenticias elaboradas; ceras animales o vegetales". Para el comercio extra-UE, de nuevo el 15 y el "21. Preparaciones alimenticias diversas".

La elección del capítulo 15 en el comercio intra-UE, se justifica al presentar la mejor penetración real, ser el tercer sector aportando al crecimiento de las exportaciones en el periodo estudiado, el más dinámico, segundo en volumen de exportaciones intra-UE y el tercero que más incrementa su saldo comercial. Para el comercio extra-UE, ocupa el primer 
lugar en penetración real, EC, ED, peso en las exportaciones, aportación al crecimiento de las mismas y mejora del saldo comercial.

El capítulo 8, en el marco intra-UE, es cuarto en penetración real, primero en competitividad, segundo en dinamismo, tercero en volumen de exportaciones y el segundo que más incrementa el saldo comercial.

El capítulo 21 es el de mejor comportamiento tras el 15, ya que es segundo en competitividad, penetración real, aportación al incremento de las exportaciones y mejora del saldo comercial; aunque su peso en el comercio extra-UE andaluz es muy pequeño.

Es necesario reseñar, que el número de partidas arancelarias reflejadas en este breve análisis es menor que las que componen cada capítulo, puesto que en algunos casos no hay datos para el año 2006, o para el 2014, o para ambos.

Los cuatro sectores escogidos son sectores de especialización y tipo 1, a excepción del 15 intra-UE.

\section{CUADRO 13: A RESUMEN DEL COMPORTAMIENTO DE LOS CAPÍTULOS DESAGREGADOS}

\begin{tabular}{|c|c|c|c|c|c|c|c|c|c|c|c|c|c|c|c|}
\hline & \multirow{2}{*}{$\begin{array}{l}\text { Var } \\
\text { IES }\end{array}$} & \multirow{2}{*}{\multicolumn{2}{|c|}{$\begin{array}{cc}\text { IEE. } & \text { Var. } \\
& P R\end{array}$}} & \multicolumn{3}{|c|}{ Тіро 1} & \multicolumn{3}{|c|}{ Тіро 2} & \multicolumn{3}{|c|}{ Tіро3 } & \multicolumn{3}{|c|}{ Tipo4 } \\
\hline & & & & $\mathrm{N}^{\mathrm{o}}$ & $\mathrm{EC}$ & ED & $\mathrm{N}^{\mathrm{o}}$ & $\mathrm{EC}$ & ED & $\mathrm{N}^{\mathrm{o}}$ & $\mathrm{EC}$ & ED & $\mathrm{N}^{\mathrm{o}}$ & $\mathrm{EC}$ & ED \\
\hline 8 & 7 & 12 & 9 & 8 & 254,6 & 226,1 & 4 & 59,8 & 47,1 & 1 & 0,9 & $-0,4$ & 1 & $-16,20$ & -16 \\
\hline 15 & 6 & 6 & 7 & 4 & 247,8 & 71,4 & 4 & $-21,9$ & 30,4 & 3 & 28,7 & $-3,5$ & 1 & $-0,13$ & 1 \\
\hline $15^{\mathrm{EX}}$ & 13 & 9 & 13 & 6 & 20,8 & 7,8 & 4 & -3.0 & 2,2 & 5 & 262,5 & -9 & 1 & 1 & 1 \\
\hline 21 & 2 & 1 & 2 & 2 & 120,2 & 0,1 & 2 & $-2,9$ & 1,4 & 0 & & & 2 & 2 & 1 \\
\hline
\end{tabular}

Var. IEE: Número de subsectores que mejoran IEE.

IEE: Número de subsectores de especialización.

Var. PR: Número de subsectores que aumentan la penetración Real.

Para cada tipo de subsector, $\mathrm{n}^{\circ}$, indica el número de partidas de dicho tipo. EC y ED indica, en millones de euros, la aportación a las exportaciones del conjunto de partidas de cada Tipo.

1 Los datos son inferiores a 12.000 euros. 2. -17.000 euros.

Fuente: Elaboración Propia a partir de los datos de ICEX (2015 b) y EUROSTAT (2015)

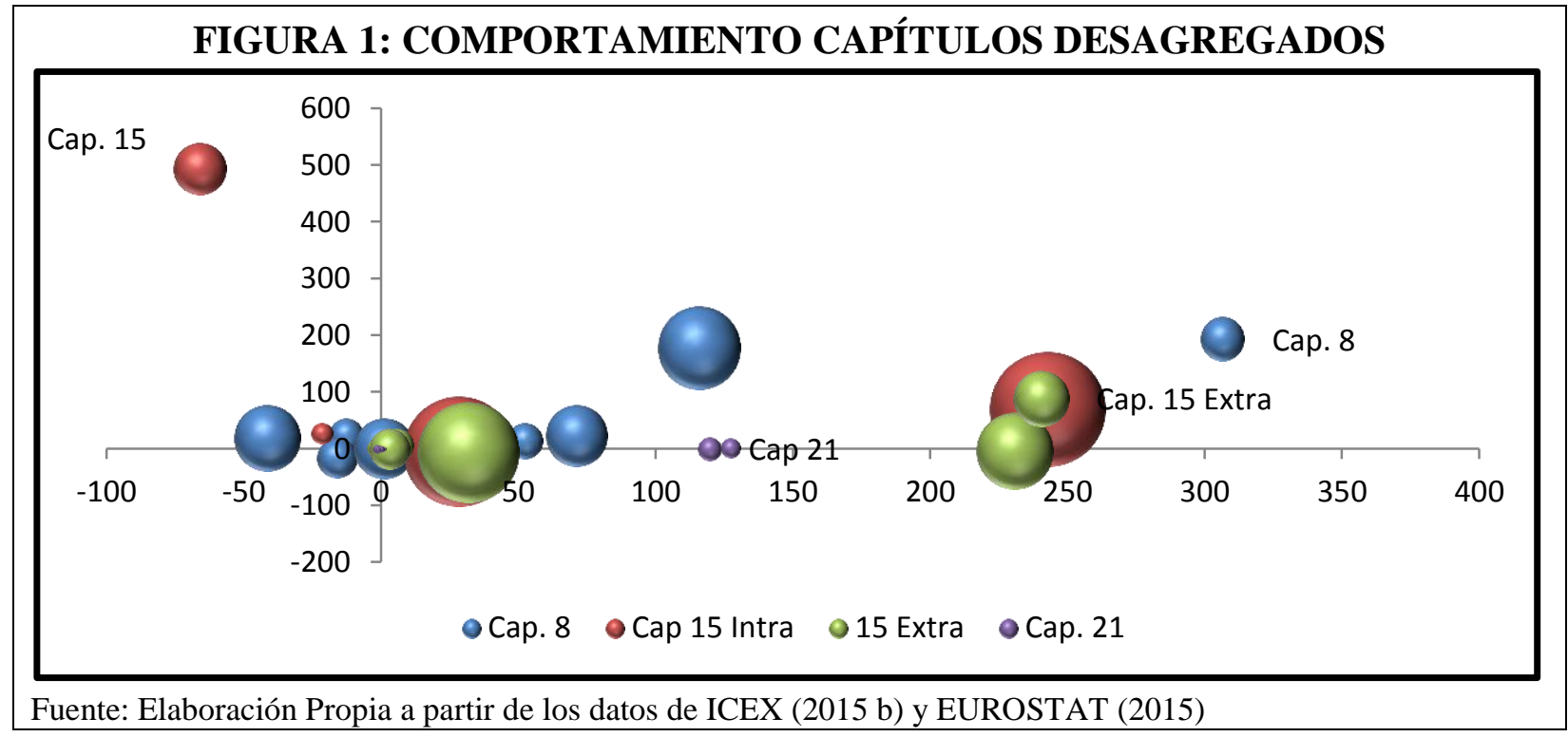


Tal como podemos deducir a partir de los datos del cuadro 13, el comportamiento de un capítulo, y los subsectores que lo componen no es homogéneo. Así, en el capítulo 8 la mayor parte de los subsectores se comportan en cuanto a la especialización, competitividad y dinamismo, como el agregado. No sucede así con el resto de sectores desglosados.

Por otro lado, la desagregación de los sectores exportadores nos ha permitido observar, que el comportamiento del sector se ve determinado por un número pequeño de subsectores, tal como podemos ver en la figura 1, donde hemos representado al unísono EC y ED y el IEE para el año 2014, tanto de los cuatro sectores seleccionados, como de los subsectores que lo componen. Cuanto más cercanas estén las burbujas que representan al subsector de la del sector, mayor es la influencia del subsector en la evolución del dinamismo y la competitividad del sector agregado. Podemos observar como un porcentaje importante de subsectores se aleja del comportamiento del capítulo al que pertenece.

Si la situación descrita ocurriera en un número mayor de capítulos, deberemos realizar análisis más desagregados, para poder obtener información más ajustada a la realidad y poder así realizar un mejor diagnostico.

\section{CONCLUSIONES}

El objetivo planteado era conocer el comportamiento competitivo del sector exportador agroalimentario andaluz, determinando dónde se ha producido la evolución más favorable de las ventas al exterior de los mismos. Las exportaciones agroalimentarias crecieron en la Comunidad Autónoma de Andalucía en el periodo analizado por valor de 3.060 millones de euros, de los cuales 2.039 corresponden a exportaciones intra-UE y 1.021 millones a las extraUE, lo que supone casi un 30 por 100 del incremento del conjunto de las exportaciones de bienes andaluces.

A lo largo del periodo la actividad exportadora agroalimentaria mantiene como base los mismos sectores de especialización sobre la que se apoyan sus ventas al exterior, con mayor variación en el caso del comercio extra-UE. Si tenemos en cuenta que una parte importante de los sectores exportadores son tipo 1, aquellos en los que ambos efectos son positivos, podemos afirmar que el sector agroalimentario andaluz ha tenido un buen comportamiento competitivo.

Como es de esperar, dada la situación de inestabilidad económica que ha mostrado la UE durante el periodo, este buen comportamiento ha sido de mayor intensidad en las exportaciones extra-UE donde el número de sectores tipo 1 de especialización es mayor que en el caso de la intra-UE, obteniendo además una mejora de la cuota de mercado en casi todos los sectores. Consecuencia del mayor dinamismo del comercio internacional fuera de la UE, las exportaciones extra-UE han ganado en importancia en el conjunto de las ventas exteriores andaluzas, con un incremento de las ventas del 115 por 100 frente al 53 por 100 de las intraEU. No obstante, la realidad del mercado único, sin fronteras y con unos procedimientos de armonización técnica y de calidad cada vez más homogéneos, sigue marcando una clara prioridad a favor de las intra-UE siguen representando algo más del 75 por 100 de las exportaciones de estos productos.

Los sectores con especialización, tanto en el comercio intra como en el extra-UE, no alcanzan ni a la mitad de los mismos y el porcentaje de exportaciones que representan está en torno al 40 por 100 en el primer caso y el 14 por 100 en el segundo. Así, en el ámbito extraUE trece sectores mejoran la especialización y ganan cuota de mercado, frente a los cinco del intra-UE. 
El número de sectores de especialización tipo 1 del comercio intra-UE es bajo, pero supone el 62,1 por 100 de las ventas de productos agroalimentarios. Son pocos, pero dado los altos valores de la especialización, podemos pensar que en el futuro se mantendrán, toda vez que el capítulo 15, no es tipo 1.

En el comercio extra-UE los siete sectores tipo 1 de especialización suponen el 62,6 por 100 de las ventas correspondientes a productos agroalimentarios, mediatizado por el capitulo 15 que alcanza los 725 millones de euros en sus ventas extra-UE. Todos ellos aumentan su especialización y ganan cuota de mercado, por lo que podemos pensar en ellos como el soporte del sector.

Aun cuando son muy pocos los sectores desglosados, el elevado número de subsectores tenían un comportamiento individual diferenciado al del sector, nos obliga a plantearnos en futuros trabajos a trabajar con partidas arancelarias y no con capítulos.

Esperamos que el análisis de concentración sectorial de las exportaciones agroalimentarias, a través del Índice de Herfindahl, y la medición de la variable latente penetración internacional del sector agroalimentario andaluz en base a diferentes ítems nos permitirá concluir en breve y con mayor grado de acierto sobre el verdadero impacto que tiene el sector en Andalucía en torno a sus exportaciones.

\section{BIBLIOGRAFÍA}

Alcántara Escolano, V. y Blanes Cristóbal, J.V. (2000): "Efectos explicativos de las exportaciones de la UE a los países de Europa central y oriental: metodología y primeros resultados", Información Comercial Española, ICE: Revista de Economía, no 786, pp. 183-190.

Coughlin, C.C. y Pollard, P. S. (2001): "Comparing Manufacturing Export Growth Across States: What Accounts for the Differences?" Federal Reserve Bank of St. Louis Review, enero / febrero 2001, pp. 25-40, recuperado de https://research.stlouisfed.org/publications/review/2001/01/01

Donoso Donoso, V. y Martín Barroso, V. (2007): "Mercados de exportación de España: presente y futuro", Información Comercial Española, ICE: Revista de Economía, no 838, pp. 41-58.

Esteve Pérez, S., Minondo Uribe-Etxeberria, A., Pallardó López, V. y Requena Silvente, F. (2009): "Análisis de competitividad de las exportaciones: Un nuevo enfoque Shift Share”, Boletín Económico de Información Comercial Española, n 2.979, pp. 13-22.

Fernández Núñez, M. T., y Márquez Paniagua, M. Á. (2009): “Análisis de la capacidad competitiva relativa de las exportaciones Intra-UE de productos agroalimentarios: el caso de la Unión Europea (UE12)". Información Comercial Española, ICE: Revista de Economía, no 851, pp. 135-156.

Fuentes Pascual, R e Hidalgo Moratal, M. (coordinadores) (1995): Problemas de Economía Aplicada, Ed. Piramide, Madrid.

Green, R. y Allaway, A. (1985): "Identification of Export Opportunities: A Shift-Share Approach", Journal of Marketing, $\quad \mathrm{n}^{\circ} 49, \quad$ pp. 83-88, recuperado de http://www.jstor.org/stable/1251178.

Gutiérrez Fernández, A. (Director) (2012): La Competitividad e Internalización de la Economía Andaluza y de sus provincias y el nuevo modelo de crecimiento, Informe $n^{o} 1$, Ed. Instituto de Estudios Cajasol, Sevilla. 
Gutiérrez Fernández, A. (Coordinador) (2014): Informe sobre la internacionalización de la Economía Andaluza y de sus provincias y el nuevo modelo productivo, Informe $n^{o} 2$, Ed. Red de Impresión, Sevilla.

Gutiérrez Fernández, A., Morán Álvarez, J.C. y Fuentes Saguar, P. (1997): "El sector exterior español y andaluz y la competitividad". Ponencia en el I Congreso de Ciencias Regionales de Andalucía: Andalucía en el umbral del siglo XX, 562-581.

ICEX - España Exportación e Inversiones (2015 a): Perfil de los exportadores españoles. Andalucía. Sectores económicos: 1. 2010 y 2014.

ICEX - España Exportación e Inversiones (2015 b): Base de Datos ESTACOM.

IECA - Instituto de Estadística y Cartografía de Andalucía (2013): Andalucía Datos Básicos 2013, recuperado de: www.juntadeandalucia.es/institutodeestadisticaycartografia/dtbas13/ADB2013.pdf.

IECA - Instituto de Estadística y Cartografía de Andalucía (2015): Contabilidad Regional de Andalucía, Base 2010, serie 1995/2013, recuperado de www.juntadeandalucia.es/institutodeestadisticaycartografia.

EUROSTAT (2015): Comisión Europea. Base de Datos de Comercio Internacional, EU Trade since 1988 by HS2 - HS4 (DS 016894), Luxemburgo.

Martín Guzmán, Ma P y Martín Pliego, FJ (1993): Curso Básico de Estadística Económica, Ed. AC, Madrid.

Myro Sánchez R., Álvarez López, E., Fernández-Otheo, C.M., Rodríguez Rod'riguez, D., Vega Crespo, J. (2013) : Fortalezas competitivas y sectores clave en la exportación española, Ed. Instituto de Estudios Económicos, Madrid

Proyecto C-Intereg. (2015 a): Base de Datos trimestral del Comercio Interregional de Bienes C-Intereg. Recuperado de http://www.c-intereg.es/C-intereg_trimestral_12_2015.zip.

Proyecto C-Intereg. (2015 b): Base de Datos de Comercio Interregional. Análisis Estadístico. Comunidades Autónomas y Ramas de Actividad. Recuperado de http://www.cintereg.es/docs/inf_complementaria/Andalucia.pdf.

Proyecto C-Intereg. (2015 c): Base de Datos de Comercio Interregional. Análisis Estadístico. Comunidades Autónomas y Ramas de Actividad, recuperado de http://www.cintereg.es/docs/inf_complementaria/R1-Agricultura_silvicultura_pesca.pdf.

Rodríguez Nuño, V. (2001): "Evolución de la producción de los sectores manufactureros en la UE. Análisis shift - share 1980-1995”, Boletín Económico de Información Comercial Española, no 2.710, pp. 9-18.

Ruiz Chico, J., Peña Sánchez, A. R. y Jiménez García, M. (2014): "Estudio de las exportaciones agroalimentarias españolas. Un análisis de competitividad a nivel regional", Colección Documentos de trabajo, $\mathrm{n}^{\circ}$ 002/2014, pp. 9-49, recuperado de http://www.centrodeestudiosandaluces.info/PDFS/DT022014.pdf.

Seguí Esquivias, C. y Montserrat Solé, A. (2008): "Evolución de la exportación catalana y composición de su crecimiento 1995/2005", Boletín Económico de Información Comercial Española, nº 2.952 , pp. 47-60. 